\title{
Pregnant and Breastfeeding Women: Drinking for Two?
}

\author{
Saptawati Bardosono ${ }^{a} \quad$ Clémentine Morin $^{c} \quad$ Isabelle Guelinckx ${ }^{c}$ Rizki Pohan ${ }^{b}$ \\ ${ }^{a}$ Department of Nutrition, Faculty of Medicine, Universitas Indonesia - Cipto Mangunkusumo General Hospital, and \\ ${ }^{b}$ R\&D AQUA Group, Jakarta, Indonesia; ' Department of Hydration and Health, Danone Research, Palaiseau, France
}

\author{
Keywords \\ Fluid intake - Beverages - Water intake from foods . \\ Pregnant $\cdot$ Breastfeeding $\cdot$ Hydration · Indonesia · 7-Day \\ fluid record
}

\begin{abstract}
The primary aim of this cross-sectional survey was to assess the total fluid intake (TFI; sum of drinking water and all other fluids) and the intake of water and all other types of beverages in a sample of pregnant and breastfeeding women representative of Java-Island, Indonesia. Therefore, 299 pregnant and 296 breastfeeding women completed a 7-day fluidspecific record. A secondary aim was to estimate the total water intake (TWI; sum of water from fluids and food moisture), and one 24-h recall was performed to determine water intake from food moisture. The median TFI of pregnant and breastfeeding women were 2,250 (1,800-2,800) and 2,360 $(1,954-2,968) \mathrm{mL} /$ day, respectively. The largest contributor to TFI was water (72 and $77 \%$ for pregnant/breastfeeding women, respectively). Pregnant women to the extent of $42 \%$ and $54 \%$ of breastfeeding women did not reach the adequate intake (Al) of water from fluids. In pregnant and breastfeeding women, the median water intake from foods was 592 and $613 \mathrm{~mL} /$ day, representing 21 and 20\% of TWI. Concluding that a high proportion of the pregnant and breastfeeding subjects did not reach the Al of water from fluid, it seems important to put in place actions such as providing education materials and ensuring access to safe wa-
\end{abstract}

\begin{tabular}{ll}
\hline KARGER & $\begin{array}{l}\text { ( } 2017 \text { The Author(s) } \\
\text { Published by S. Karger AG, Basel }\end{array}$ \\
$\begin{array}{l}\text { E-Mail karger@karger.com } \\
\text { www.karger.com/anm }\end{array}$ & $\begin{array}{l}\text { This article is licensed under the Creative Commons Attribution- } \\
\text { NonCommercial-NoDerivatives 4.0 International License (CC BY- } \\
\text { NC-ND) (http://www.karger.com/Services/OpenAccessLicense). } \\
\text { Usage and distribution for commercial purposes as well as any dis- } \\
\text { tribution of modified material requires written permission. }\end{array}$
\end{tabular}

ter. Moreover, future surveys should dedicate attention to the assessment of fluid intake and hydration status among pregnant and breastfeeding women in other countries.

$$
\begin{aligned}
& \text { C } 2017 \text { The Author(s) } \\
& \text { Published by S. Karger AG, Basel }
\end{aligned}
$$

\section{Introduction}

Diet quality during pregnancy and lactation exerts an influence on both the fetal or neonatal development and maternal well-being [1]. Recent research suggests that inadequate levels of maternal nutrients during pregnancy may lead to "reprogramming" within the fetal tissues that predispose the infant to chronic illnesses in adulthood [2]. Yet, in this research, the impact of total water intake (TWI; water originates from fluids and food moisture) is rarely considered. Nevertheless, health authorities such as European Food Safety Authority (EFSA) and the Institute of Medicine (IoM) state that the requirements for TWI are increased during pregnancy and breastfeeding. In 2010, EFSA set the adequate intake (AI) of TWI for pregnant women at $2,300 \mathrm{~mL} /$ day, which is an increase of $300 \mathrm{~mL} /$ day compared to that for non-pregnant women. This increase is based on the fact that the energy requirements during pregnancy increase by $300 \mathrm{kcal} /$ day. For breastfeeding women, the guideline of EFSA is to increase TWI $700 \mathrm{~mL} /$ day compared to non-breastfeeding women, resulting in an AI of 2,700 mL/day [3]. This increase 
aims to compensate the water loss through breast milk. The IoM based the dietary reference value for TWI on the median TWI observed in NHANES III, which was 3,000 $\mathrm{mL} /$ day for pregnant women and $3,800 \mathrm{~mL} /$ day for breastfeeding women [4]. Like EFSA, the Indonesian Ministry of Health set the dietary reference values based on a theoretical relationship between water intake and the energy intake, indicating that for each kcal of energy intake, 1-1.5 mL of water needs to be consumed [5]. Pregnant women are therefore advised to add $300 \mathrm{~mL} /$ day of water to the $\mathrm{AI}$ of $2.3 \mathrm{~L} /$ day recommended for non-pregnant women. Breastfeeding women are advised to increase TWI by $800 \mathrm{~mL} /$ day during the first 6 months postpartum and by $650 \mathrm{~mL} /$ day after 6 months postpartum. To the best of our knowledge, there have been no nutritional surveys among pregnant and breastfeeding women dedicated to fluid intake in Indonesia.

The largest dietary sources of TWI are fluids (sum of drinking water and all other fluid types), which contribute $70-80 \%$ of TWI $[3,4]$. Water from foods is assumed to contribute $20-30 \%$ to TWI, yet the type and quantities of foods eaten will determine this contribution. Since many factors (i.e., food availability, climate, cultural factors, health and economic status, age, psychosocial factors, religious factors and agricultural practices) influence the selection of foods [6], the amount of water obtained from food can vary between individuals and countries. Only limited data is currently available in Indonesia on the contribution of water from foods to TWI.

To address the lack of data regarding fluid intake in pregnant and breastfeeding women in Indonesia, and to evaluate whether an intervention is needed to ensure an AI of water from fluids, the primary aim of the present analysis was to assess the fluid intake of drinking water and all other fluid types in a sample of pregnant and breastfeeding women representing 3 large cities of Indonesia. Moreover, the adherence to the AI of water from fluids set by the Indonesia Ministry of Healthy was evaluated. The secondary aim was to estimate the contribution of water from foods to TWI.

\section{Materials and Methods}

This cross-sectional survey was conducted from January to April 2014, in Jakarta, Yogyakarta and Surabaya, representing the Java island of Indonesia. All households with pregnant and breastfeeding women under the selected maternity clinic in each of the study areas were eligible for recruitment. Eligible subjects were given oral and written information about the study objec- tives and protocol. If they were willing to participate, a written informed consent was obtained from them. The study was approved on December 23, 2013, by the Ethics Committee of the Faculty of Medicine Universitas Indonesia (number 783/H2.F1/ ETIK/2012). Full protocol details with the inclusion and exclusion criteria have been described elsewhere [7]. Additionally, this survey followed the same sampling method, protocol and method for fluid assessment as those performed in the Liq.In ${ }^{7}$ surveys $[8,9]$.

The screening was performed by a nutritionist during an athome visit. During this visit, details about pre-pregnancy weight and height for pregnant women and current weight and height for breastfeeding women were collected. The nutritionist gave all instructions on how to complete the first day of the 7-day fluid dairy. The next day, the nutritionist returned to the participant to collect and check for completion of the record of the previous day and provide a new record for the following day. These daily home visits allowed women to maintain a high level of participation and to avoid copying data from one day to another. Participants who did not complete all the 7 days of the specific fluid dairy, who had reported a mean daily fluid intake below $400 \mathrm{~mL} /$ day or higher than $6,000 \mathrm{~mL} /$ day were excluded from the analysis.

The 7-day fluid record was structured to collect the following detailed information on each drinking act in open spaces on the record: the hour of consumption, the type of fluid, the brand of fluid, the volume of the recipient from which the volume was consumed and the volume actually consumed. To assist the subjects in estimating the consumed volumes, the records were supported by a photographic booklet of standard containers of fluids. Only consumption of non-alcoholic beverages were recorded and were classified into drinking water (boiled water and bottled water), hot beverages (coffee and tea), milk beverages, Sugared Sweetened Beverages (SSB; carbonated sweetened drinks, still sweetened drinks [SSD], functional and flavoured beverages, and juices) and others beverages. Total fluid intake (TFI) was defined as the sum of volumes of all these categories. Congee, soup and other watery snacks were classified as foods, and not included in TFI.

To estimate water from food moisture, the nutritionist performed on the day after the 7-day fluid recording a dietary interview to assess what the participants ate during the previous $24 \mathrm{~h}$ period. It has been demonstrated that TFI assessed with a 7-day fluid diary was significantly higher, especially among individuals with a higher TFI, than TFI estimated with a $24 \mathrm{~h}$ recall. For this reason, TWI was defined as the sum of TFI on the 7th day of the 7-day fluid record and the water intake from foods estimated with the 24-h food recall. Additionally, because data from a 7-day diary is considered to be the golden standard and because TFI is the largest contributor to TWI, an AI was defined as having a 7-day mean TFI $\geq 80 \%$ of the AI for TWI set by the Ministry of Health of the Republic of Indonesia [5].

Data analysis was performed by using commercial software JMP, version 10 (SAS Campus Drive, Cary, NC, USA). Continuous variables were presented as median, 25 th and 75 th percentile as indicated for skewed variables, and dichotomous variables as percentages. Group comparisons between pregnant and breastfeeding women were assessed with the chi-square test. The median intakes are estimated values, taking into account all consumers including non-consumers. Significance level was set at $5 \%$. 


\section{Results}

The analysis was done on a sample of 595 women (299 pregnant and 296 breastfeeding). The baseline characteristics and the distribution of participants by pregnancy trimester or duration of breastfeeding are summarized in Table 1 . The mean age of the pregnant and breastfeeding women was $28.6 \pm 4.1$ years, within the range from 19 to 38 years. The mean pre-pregnancy BMI for pregnant women and the mean BMI for breastfeeding women were $25.6 \pm 4.5$ and $24.3 \pm 4.6 \mathrm{~kg} / \mathrm{m}^{2}$ respectively. Thirty-one percent of all women were overweight $\left(25-30.0 \mathrm{~kg} / \mathrm{m}^{2}\right)$ and $13 \%$ were obese $\left(\geq 30.0 \mathrm{~kg} / \mathrm{m}^{2}\right)$.

Table 2 shows the median of the 7-day mean TFI. In pregnant and breastfeeding women, the 7-day median of TFI was 2,250 and 2,360 $\mathrm{mL} /$ day, respectively, with a range from 2,220 to $2,300 \mathrm{~mL} /$ day and 2,300-2,378 mL/day. Forty-two percent of pregnant women and $54 \%$ of breastfeeding women did not reach the AI of water from fluids, as recommended by the Ministry of Health, Republic of Indonesia [5].

No significant differences in TFI were observed between trimesters of pregnancy, while women breastfeeding for at least 1 year had a lower TFI $(2,154[1,771-$ $2,737] \mathrm{mL} /$ day) than the 2 others groups of breastfeeding women $(2,607 \pm 754 \mathrm{~mL} /$ day for $0-5$ months postpartum; $p=0.005$ and $2,538 \pm 807 \mathrm{~mL} /$ day for $6-11$ months postpartum; $p=0.035$ ). There was a significant difference in TFI between the BMI classes; however, this significant difference was observed only among pregnant women $(p=0.005)$. Pregnant women with pre-pregnancy underweight had a significantly lower median TFI $(1,894 \mathrm{~mL} /$ day $[1,607-2,195 \mathrm{~mL} /$ day $]$ than women who were overweight $(2,476 \mathrm{~mL} /$ day $[1,876-3,053$ $\mathrm{mL} /$ day]; $p=0.014)$ and those with obesity $(2,356 \mathrm{~mL} /$ day $[1,940-2,853 \mathrm{~mL} /$ day]; $p=0.012)$. The observed TFI median among women with pre-pregnancy overweight was even significantly higher than that of women with normal pre-pregnancy weight (median 2,128 [1,691$2,726 \mathrm{~mL} /$ day]; $p=0.007$ ). Among pregnant women, there was no significant difference in TFI neither according to occupation nor according to educational levels $(p=0.094$ and $p=0.471$, respectively). Similar observations were made among breastfeeding women (occupation $p=0.589$; educational level $p=0.865$ ).

The median water intake from food and TWI is also presented in Table 2. No significant differences between pregnant and breastfeeding women were observed for water intake from foods $(p=0.20)$ and TWI $(p=0.18)$. The median contribution of water from foods to TWI was
Table 1. General characteristics of the Indonesian study sample of pregnant and breastfeeding women

\begin{tabular}{ccc}
\hline & $\begin{array}{l}\text { Pregnant } \\
\text { women }\end{array}$ & $\begin{array}{l}\text { Breastfeeding } \\
\text { women }\end{array}$ \\
\hline Sample size & 299 & 296 \\
1st trimester & 100 & - \\
2nd trimester & 103 & - \\
3rd trimester & 96 & - \\
0-5 months & - & 101 \\
6-11 months & - & 97 \\
12-24 months & - & 98 \\
Age, years & $28.5(4.3)$ & $28.6(4)$ \\
Body mass index, & & $24.3(4.6)$ \\
kg/m ${ }^{2}$ & $25.6(4.5)$ & \\
\hline
\end{tabular}

Data are expressed as mean (SD).

Table 2. Daily and 7-day median of total fluid intake, mean water intake from foods and total water intake

\begin{tabular}{lcc}
\hline & $\begin{array}{l}\text { Pregnant women } \\
(n=299)\end{array}$ & $\begin{array}{l}\text { Breastfeeding } \\
\text { women }(n=296)\end{array}$ \\
\hline Total fluid intake & $2,250(1,800-2,800)$ & $2,360(1,954-2,968)$ \\
Water intake from food & $592(430-772)$ & $613(436-784)$ \\
Total water intake & $2,863(2,225-3,593)$ & $2,970(2,531-3,633)$
\end{tabular}

Data are expressed as median (25th-75th percentiles).

$21 \%(15-30 \%)$ and $20 \%$ (14-27\%), respectively, for pregnant and breastfeeding women.

The contribution of each type of beverage to TFI is presented in Figure 1. Among the different fluid types, drinking water (boiled and bottled water) is the highest contributor to TFI in pregnant and breastfeeding women (72 and 77\%, respectively). Pregnant women consumed more boiled water than bottled water, whereas breastfeeding women consumed more bottled water than boiled water. The second fluid type that contributed the most to TFI was hot beverages: $10 \%$ among pregnant and $11 \%$ among breastfeeding women. Both groups drank more tea (pregnant $190 \mathrm{~mL} /$ day [range 71-323 mL/day]; breastfeeding, 254 [98-439 mL/day] than coffee (pregnant $0 \mathrm{~mL} /$ day [0-0 mL/day]; breastfeeding, 0 [0-29 mL/day]). The third contributor to TFI was SSB with a daily intake of 140 (54-274) $\mathrm{mL} /$ day for pregnant women and 57 (0189) $\mathrm{mL} /$ day for breastfeeding women. In pregnant and breastfeeding women, respectively, SSD contributed to 57 and $51 \%$ of total SSB intake. Pregnant women had a sig- 
Fig. 1. Contribution of the different types of beverages to total fluid intake (\%) in an Indonesia sample $(n=595)$ of pregnant and breastfeeding women. SSD, still sweetened drinks; CSD, carbonated sweetened drinks.

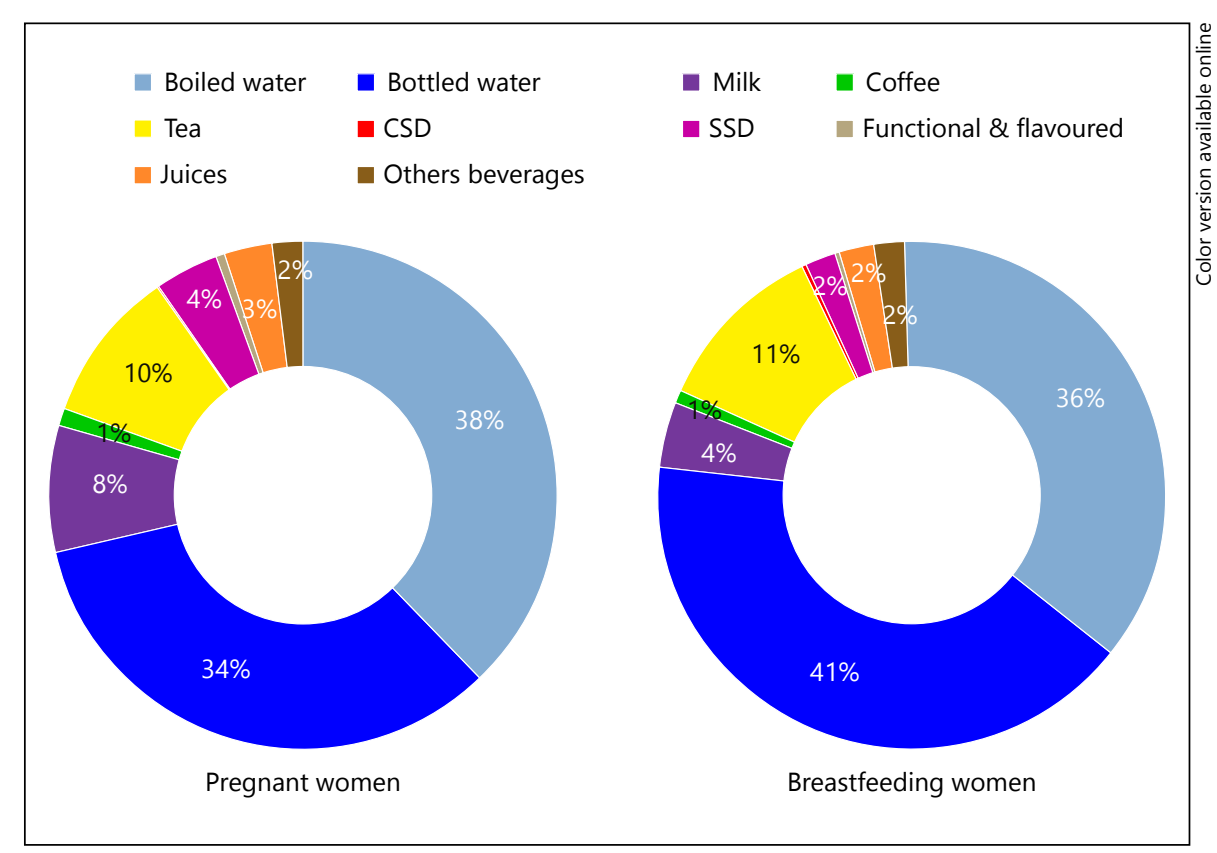

nificantly higher median intake of SSD compared to breastfeeding women $(57[0-150]$ vs. $0[0-86] \mathrm{mL} /$ day, $p<0.001)$.

\section{Discussion}

This is the first survey among pregnant and breastfeeding women in Indonesia focusing on TFI, assessing the contribution of the different fluid types to TFI, and determining the contribution of water intake coming from foods to TWI. The primary finding of this survey was that the mean TFI of pregnant and breastfeeding women was 2.3 and $2.5 \mathrm{~L} /$ day, respectively. Consequently, 42 and 54\%, respectively, of pregnant and breastfeeding women did not reach the AI of water from fluids set by the Indonesian Ministry of Health [5]. The fluid type consumed the most by both pregnant and breastfeeding women was water.

Compared to an Indonesian sample of 307 non-pregnant and non-breastfeeding women living in the same 3 cities, the median intakes of pregnant and breastfeeding women differed only by 59 and $169 \mathrm{~mL} /$ day, respectively. This increase is considerably less than the recommended increase of 300 and $800 \mathrm{~mL} /$ day for pregnant and lactating women [5]. Due to the absence of hydration biomarkers, no conclusion can be drawn whether or not this increase of TFI covers their actual requirements of TWI.
The data addressing the secondary aim of the present analysis indicated that the contribution of water from foods to TWI is $21 \%(15-30 \%)$ and $20 \%(14-27 \%)$ in pregnant and breastfeeding women. These results are consistent with the EFSA reference values for TWI that take into account the $20 \%$ of TWI from food [3]. A previous study performed in Greece [10] observed a higher contribution of water from foods (25\%) among pregnant women than the findings of this analysis. However, the contribution observed among a non-pregnant population in Greece was comparable to our finding (15 and $24 \%$ water from foods contributed to TWI in summer and winter respectively).

Several limitations of this survey need to be considered. First, these results cannot be extrapolated to all Indonesian pregnant and breastfeeding women because data collection was performed only in the Java Island. Furthermore, data collection was performed during a particular period of the year and therefore, seasonality was not taken into account. However, since the temperature is rather stable on the Java Island across the year, the effect of seasonality is not taken into account. Additionally, the estimation of water from food was performed with a single $24 \mathrm{~h}$ recall, which might result in limited representation of the habitual weekly food intake. The comparison between the pregnancy trimesters and postpartum periods needs to be considered with caution, as this survey was cross-sectional and not a longitudinal sur- 
vey, following the intake of the participant from the prepregnancy period into pregnancy and the postpartum period.

Despite these limitations, this survey contributes new valuable information to the literature on fluid intake of a specific vulnerable target population. Given that TFI was assessed using a 7-day fluid specific record, which is considered the reference method in nutritional assessment [11], and that a photographic booklet of standard fluid containers was provided to the participants, the accuracy of the data is considered to be high. The daily visit of the nutritionist reduced the risk of non/incomplete recording of the record as illustrated by the high compliance rate (i.e., >95\%) of the survey. Moreover, the survey was performed among a large sample, with an equal distribution over pregnancy trimester and the postpartum period.

In summary, this survey indicated that a large proportion of this Indonesia pregnant and breastfeeding sample had an in AI of water from fluids. This finding implies that actions from midwives, general practitioners and others doctors are appropriate enough to assist these women towards an AI of water. Such actions could be to provide education regarding the required intake of water, or to ensure availability of safe water. These actions were identified as being key to ensure efficacy of a water intervention [12-14]. Moreover, urine color has been demonstrated to be a valid marker of hydration status in pregnant and breastfeeding women [15]. So providing a urine color chart could support both actions previously mentioned. The potential efficacy of these actions is high, since pregnancy and breastfeeding are especially powerful "teachable moments" for the promotion of healthy behaviors: many women are concerned about the health of their babies during pregnancy and are in frequent contact with their healthcare providers [16].

\section{Acknowledgements}

The study was funded by a research grant from PT Tirta Investama (Danone Aqua). The costs to publish in open access were covered by Danone Research.

\section{Disclosure Statement}

I.G. and C.M. are employees of Danone Research. R.P. is an employee of R\&D AQUA Group. S.B. has no conflict of interest to declare.

\section{References}

1 Fowles ER: What's a pregnant woman to eat? A review of current USDA dietary guidelines and MyPyramid. J Perinat Educ 2006;15:2833.

2 Godfrey KM, Barker DJ: Fetal programming and adult health. Public Health Nutr 2001;4: 611-624.

3 European Food Safety Authority Panel on Dietetic Products Nutrition and Allergies (NDA): Scientific opinion on dietary reference values for water. EFSA J 2010;8:1459_ 1507.

4 Institute of Medicine, Food and Nutrition Board: Dietary Reference Intakes for Water, Potassium, Sodium, Chloride and Sulfate. Washington, National Academies Press, 2004.

5 Ministry of Health of the Republic of Indonesia: Recommended Nutritional Intake for Indonesian Population. Jakarta, Ministry of Health, 2013.

6 Mela DJ: Food choice and intake: the human factor. Proc Nutr Soc 1999;58:513-521.

7 Bardosono S, Prasmusinto D, Hadiati DR, Purwaka BT, Morin C, Pohan R, Sunardi D,
Chandra DN, Guelinckx I: Fluid intake of pregnant and breastfeeding women in Indonesia: a cross-sectional survey with a seven-day fluid specific record. Nutrients 2016;8: pii:E651.

8 Ferreira-Pego C, Guelinckx I, Moreno LA, Kavouras SA, Gandy J, Martinez H, et al: Total fluid intake and its determinants: crosssectional surveys among adults in 13 countries worldwide. Eur J Nutr 2015;54(suppl 2):3543.

9 Guelinckx I, Ferreira-Pego C, Moreno LA, Kavouras SA, Gandy J, Martinez H, et al: Intake of water and different beverages in adults across 13 countries. Eur J Nutr 2015;54(suppl 2):4555.

10 Malisova O, Protopappas A, Nyktari A, Bountziouka V, Antsaklis A, Zampelas A, Kapsokefalou M: Estimations of water balance after validating and administering the water balance questionnaire in pregnant women. Int J Food Sci Nutr 2014;65:280285.

11 Coulston AM, Boushey CJ, Ferruzzi MG: Dietary Assessment Methodology. Nutrition in the Prevention and Treatment of Disease. Amsterdam, Academic Press, 2008, pp 1-44.

12 Gomez P, Boesen-Mariani S, Lambert JL, Monrozier R: A water intervention program to improve fluid intakes among French women. Nutr Today 2013;48:S40-S42.

13 Lahlou S, Boesen-Mariani S, Franks B, Guelinckx I: Increasing water intake of children and parents in the family setting: a randomized, controlled intervention using installation theory. Ann Nutr Metab 2015; 66(suppl 3):26-30.

14 Storckdieck Gennant Bonsmann S, Mak NT, Louro Caldeira S, Wollgast J: How to Promote Water Intake in Schools: A Toolkit. Luxembourg, Publications Office of the European Union, 2016.

15 McKenzie AL, Munoz CX, Ellis LA, Perrier ET, Guelinckx I, Klein A, Kavouras SA, Armstrong LE: Urine color as an indicator of urine concentration in pregnant and lactating women. Eur J Nutr 2017;56:355-362.

16 Phelan S: Pregnancy: a "teachable moment" for weight control and obesity prevention. Am J Obstet Gynecol 2010;202:135.e1-e8. 